\title{
Freezing in the bulk controlled by prefreezing at a surface
}

\author{
A. J. Page and R. P. Sear \\ Department of Physics, University of Surrey, Guildford, Surrey GU2 7XH, United Kingdom \\ (Received 8 April 2009; revised manuscript received 4 June 2009; published 18 September 2009)
}

\begin{abstract}
We use Monte Carlo simulations of the Lennard-Jones model to study the nucleation of a crystal phase at a flat surface. Our motivation is the observation that crystal phases almost always nucleate at a surface. We find that a surface phase transition (prefreezing) can control nucleation of the bulk crystal. This finding should be general and so surface phase behavior should be considered whenever nucleation of bulk phases at surfaces is considered. Also, nucleation of the bulk crystal transforms smoothly into the nucleation of a surface crystal layer as the bulk transition is crossed.
\end{abstract}

DOI: 10.1103/PhysRevE.80.031605

PACS number(s): 64.60.qe, 61.30.Hn

\section{INTRODUCTION}

Crystallization is a common but poorly understood phenomenon. This is despite the fact that understanding it is vital in fields from earth science and condensed-matter physics, through to pharmaceuticals. Crystallization almost always starts with the new crystal forming at a surface; this is called heterogeneous nucleation [1]. Thus, any change at the surface will affect nucleation of the bulk crystal. Here, we study nucleation at a surface in a simple model of a noble gas. We find that for strongly attractive surfaces, freezing can actually occur above the melting point. This is a surface phase transition called prefreezing, and when it has occurred it abolishes the nucleation barrier to bulk freezing. Liquids in contact with the surface cannot be supercooled. We find that for sufficiently strongly attractive surfaces, prefreezing is at most a very weakly first-order transition and so there is little or no hysteresis associated with it, and hence with bulk freezing. For less strongly attractive surfaces, there are substantial barriers to prefreezing and the liquid may be supercooled with respect to the bulk transition as well as the surface transition. We observe substantial barriers even when the bulk crystal wets the surface-liquid interface contradicts classical nucleation theory $[1,2]$, which predicts that the nucleation barrier disappears at the wetting transition.

Our final observation is that nucleation of the surface crystal phase just above the melting temperature, is indistinguishable from nucleation of the bulk crystal just below the melting temperature. This is despite the fundamentally different nature of surface and bulk phases.

The most closely related earlier work has been on hardsphere-like colloidal particles at a hard wall, and on freezing in porous media. The hard-sphere crystal wets the hard-wallhard-sphere-fluid interface $[3,4]$. In addition, hysteresis associated with a surface transition has been observed by Dijkstra [3], and nucleation of the bulk crystal phase studied by Auer and Frenkel [5]. Together, the work of these authors implies that there is a nucleation barrier for hard spheres at a hard wall despite the fact that the crystal phase wets the wall. We find the same behavior in our Lennard-Jones model. Gubbins and co-workers [6-9] have studied freezing in slit pores, concentrating on a model of methane in slit pores with graphite surfaces. They found prefreezing of surface layers in these pores. Our results are in good agreement with their results.

\section{SIMULATION DETAILS}

We study the crystallization of Lennard-Jones particles at a surface that attracts the particles. The interaction potential, $U_{W P}$, between the surface and the particles is given by

$$
U_{W P}=\epsilon_{W S}\left[(\sigma / z)^{9}-(\sigma / z)^{3}\right]
$$

for $z$ the distance between the particle and the surface, $\sigma$ the diameter of a particle and $\epsilon_{W S}$ the strength of the wallparticle interaction. The well depth for the attraction to the surface is $-0.385 \epsilon_{W S}$. The Lennard-Jones potential between particles is truncated and shifted at $2.5 \sigma$ whereas the wallparticle potential is not truncated. We work at reduced temperatures $T^{*}=k T / \epsilon$, where $\epsilon$ is the well depth of the LennardJones potential. Our Metropolis Monte Carlo simulations [10] are all performed in the canonical ensemble in a cubic simulation box with side $L$. A soft repulsive wall is placed opposite the attractive one and periodic boundary conditions are applied to the other two directions.

As in earlier work [11], we use bond order parameters to identify the crystalline particles [11-15]. The only difference between here and Ref. [11] is a change in how many crystal "links" a particle must have before it is counted as crystalline. In this change we follow the surface nucleation work of Mendez-Villuendas et al. [14]. Instead of requiring that a particle has 5 or more crystal "links," we require that these links exist between it and at least 55\% of its nearest neighbors, and that it has at least 4 nearest neighbors. This is better able to distinguish crystalline and liquid particles at a surface where the coordination number is lower than in the bulk.

As we will see, nucleation of the bulk crystal is dramatically affected by surface phase behavior. Thus we will first determine the surface phase behavior at a surface in contact with a liquid phase near the triple-point temperature, $T_{T P}^{*}$ $=0.65[11]$. This is the temperature at which the vapor, liquid and crystal phases coexist. The Lennard-Jones model shows surface melting at the triple point, i.e., the liquid phase wets the crystal-vapor interface [16].

\section{SURFACE PHASE BEHAVIOR}

To estimate the surface phase behavior we performed a number of direct simulations at a fixed temperature $T^{*}$ $\geq T_{T P}^{*}$. The reduced wall strength $\epsilon_{W S} / \epsilon$ is varied throughout 


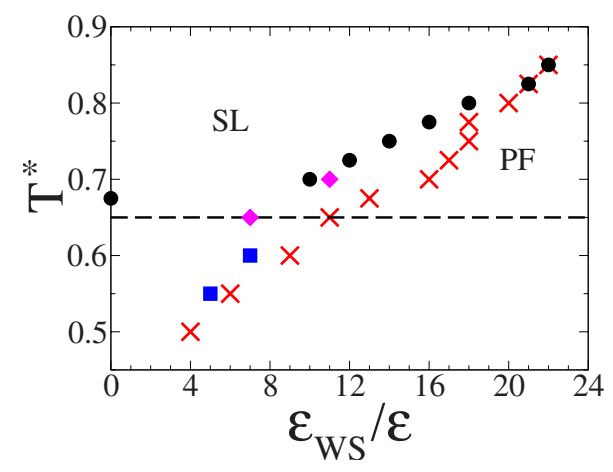

FIG. 1. (Color online) This figure summarizes our results for both surface phase behavior and nucleation. The red crosses and black circles are where crystallization occurs on increasing $\epsilon_{W S}$, and melting on decreasing $\epsilon_{W S}$, respectively. The dashed horizontal line denotes the triple point at $T_{T P}^{*}$. Above this horizontal line we have prefreezing $(\mathrm{PF})$ and the red crosses mark where a surface crystal phase appears. Below the horizontal line the red crosses mark where the bulk crystal rapidly nucleates. The black circles mark where the surface crystal phase melts into the surface liquid (SL). For $T^{*}=0.65$ and $T^{*}=0.7$ the positions of the equilibrium prefreezing wall transitions have been estimated and marked on as pink diamonds. Below $T_{T P}^{*}$ and above the line of red crosses bulk crystallization is an activated process; blue squares mark where nucleation rates were calculated.

the simulation. Initially the reduced wall strength $\epsilon_{W S} / \epsilon=5$, it is then increased by steps of 1 . After each step the system is run for $3.5 \times 10^{4}$ cycles. A cycle is one trial move per particle. We did this until we observed surface freezing. Above $T_{T P}^{*}$ we found a prefreezing transition, where a limited number of crystalline layers formed at the surface. The surface transition is called prefreezing as it occurs before freezing, i.e., at higher temperatures. The point at which surface freezing occurs is plotted as a red cross in Fig. 1. Melting of an existing surface crystalline layer is studied in a similar manner, except that the reduced wall strength is reduced in steps of 1 . The point where we observe melting is plotted as a black circle in Fig. 1. The black circles and red crosses provide lower and upper bounds, respectively, for the position of the prefreezing transition. These simulations are performed in a simulation box with $L=15 \sigma$ and the results are subject to a $\pm 1 \epsilon_{W S} / \epsilon$ error. To test for finite size effects a number of fixed temperature, fixed $\epsilon_{W S} / \epsilon$ simulations are performed in an $L=35 \sigma$ simulation box. The results are consistent with Fig. 1 and show no systematic differences.

Near the triple point we observe substantial hysteresis on varying $\epsilon_{W S}$, i.e., the black circles and red crosses are far apart. At the triple-point itself prefreezing becomes wetting. This defines a wall strength $\epsilon_{W S}^{(w e t)} / \epsilon$ above which the crystal phase wets the interface between the surface and the liquid phase. In order to obtain a better estimate of $\epsilon_{W S}^{(w e t)} / \epsilon$, we reduce hysteresis on freezing by introducing a crystalline seed at the surface [17]. In order to get as close to the wetting wall strength as possible we use a large simulation box with $L=35 \sigma$ containing a large crystal seed. The seed used was a parallelepiped of two $\{111\}$ planes in the plane of the surface and stacked one on top of the other. The first layer consisted of $21 \times 10$ particles with a second layer of $21 \times 9$ particles.

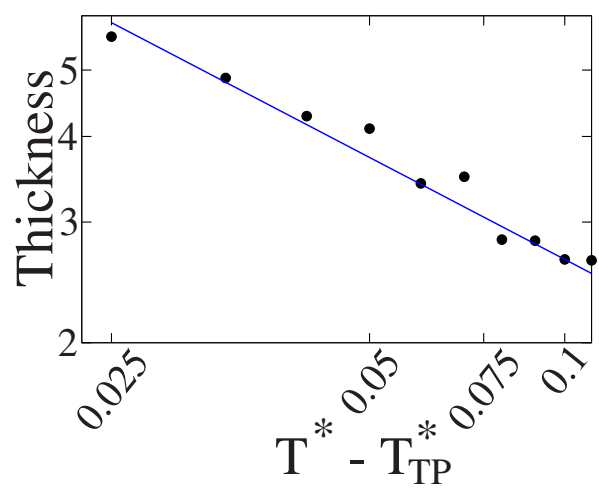

FIG. 2. (Color online) A plot on a double logarithmic scale of the thickness against $T^{*}-T_{T P}^{*}$, for a surface with $\epsilon_{W S} / \epsilon=20$. The simulation data are plotted as black circles, and the blue line is a fit by a function of the form Thickness $=A\left(T^{*}-T_{T P}^{*}\right)^{B}$ with $A=0.85$ and $B=-0.49$.

In the presence of a crystal seed the crystal phase forms for $\epsilon_{W S} / \epsilon \geq 7$. No nucleation is found at $\epsilon_{W S} / \epsilon=6$. Thus, we estimate that wetting by the crystal phase occurs at $\epsilon_{W S}^{(w e t)} / \epsilon$ $\simeq 7$.

Our finding of substantial hysteresis near the bulk transition at the triple point is consistent with experiments on liquid wetting films by Bonn et al. [18]. They found that the nucleation barrier to evaporation of a thick liquid wetting layer was large and we find a large barrier on melting of a thick wetting layer of the crystal. As the temperature is increased away from $T_{T P}^{*}$, the thickness of the surface crystal decreases and so does the hysteresis. This suggests the transition is becoming more weakly first order. We find that above $T^{*}=0.8$ the hysteresis is too small to measure.

Our surface has a long-range, $z^{-3}$, attraction for the particles. Thus, as we move away from bulk coexistence at the triple point the thickness of the wetting layer is expected to vary as $\left(T^{*}-T_{T P}^{*}\right)^{-1 / 3}$ [19]. In Fig. 2 we have plotted the thickness of the surface crystalline layer as a function of distance from the triple point. The thickness is estimated by dividing the number of crystalline particles by the total number of particles in the first layer. We see that the thickness of the surface crystalline layer rapidly increases as the triple point is approached. However, the apparent exponent is close to $-1 / 2$ not $-1 / 3$. It should be borne in mind that we can study only a limited range of thicknesses in our relatively small systems.

Far from the triple point, the surface crystal phase is thin, $\approx 2$ layers thick (see Fig. 3(b)). Here, freezing appears to be either continuous or close to continuous. The freezing does not seem to occur via the growth from a single nucleus. As the system is supercooled with respect to the prefreezing temperature for a particular wall strength, the number of small crystallites present at the surface grows. Figure 4(a) shows a metastable configuration for $T^{*}=0.7$ at $\epsilon_{W S} / \epsilon=14$. Here, formation of a prefreezing layer would lower the free energy but no crystallization is observed in a long direct run of $10^{6}$ cycles. At $\epsilon_{W S} / \epsilon=15$ crystallization takes place via the coalescence of precritical nuclei to form a post-critical nucleus. This mechanism was also observed by Zhang and Lui [20] in their work on colloidal systems at a wall. 


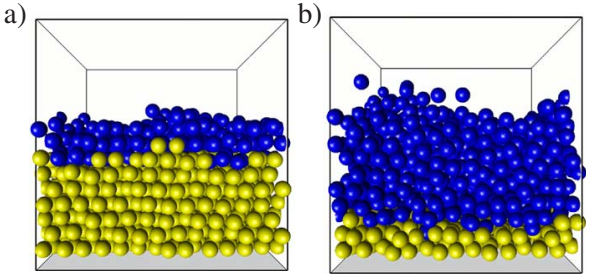

FIG. 3. (Color online) Simulation snapshots of a surface crystal at (a) $T^{*}=0.65$ and (b) $T^{*}=0.75$. In (a) $\epsilon_{W S} / \epsilon=26$, the wall interaction is greater than $\epsilon_{W S}^{(w e t)} / \epsilon$ and the surface crystal phase wets the liquid-wall interface. The liquid layer at the crystal-vapor interface is due to surface melting. For $T^{*}>T_{T P}^{*}$, at sufficiently attractive walls a prefreezing layer is formed. (b) shows such a prefreezing layer at a wall interacting with $\epsilon_{W S} / \epsilon=20$. Crystalline particles are shown in yellow and particles in a liquidlike environment are shown in dark blue.

Prefreezing, as a surface phase transition, is a transition in two dimensions. It is known that freezing in two dimensions can (but does not have to) occur via a continuous mechanism that is qualitatively different from the mechanism of freezing in three dimensions. This mechanism is called the KosterlitzThouless-Halperin-Nelson-Young (KTHNY) [21] mechanism. We find that the transition appears to be more and more weakly first order as we move away from the triple point and the surface crystal phase becomes thinner and more two dimensional. However, our system sizes and run lengths are too small to determine whether prefreezing simply becomes a very weakly first-order transition, or whether it actually occurs via the KTHNY mechanism sufficiently far from the triple point.

Gubbins and co-workers [6-9] have studied a system that is similar to ours, except that they studied not a single sur- a)

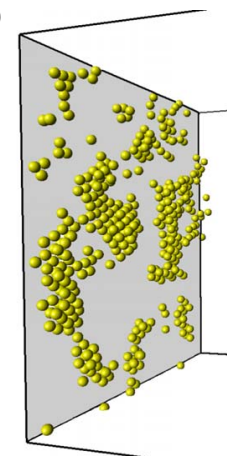

b)

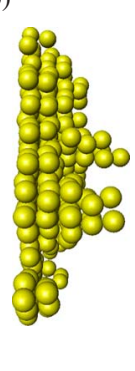

c)

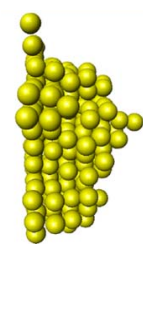

FIG. 4. (Color online) Simulation snapshots. Unlike in Fig. 3 in these snapshots only crystalline particles are shown, the particles in liquidlike environments are not shown. (a) is a snapshot of a wall at $T^{*}=0.7$ and $\epsilon_{W S} / \epsilon=14$, where the equilibrium surface state is with a crystalline layer. However, the snapshot is of the metastable surface liquid. Under these conditions the relatively large surface crystallites seen in the snapshot fluctuate in and out of existence. (b) and (c) are snapshots of near-critical nuclei, i.e., nuclei near the top of the barrier. They are obtained from FFS simulations at (b) $T^{*}=0.6$, $\epsilon_{W S} / \epsilon=7$, and (c) $T^{*}=0.55, \epsilon_{W S} / \epsilon=5$. In each case only the crystalline particles that form the largest cluster are shown. These clusters are of 318 and 213 particles, respectively. In both cases the nucleus forms with a $\{111\}$ crystal plane in contact with the wall. (a) is for a box with sides $L=35 \sigma$ long while in (b) and (c) $L=25 \sigma$. face as we do but a slit pore. Our and their results are consistent, compare our Fig. 1 with Fig. 4 of Ref. [9]. They too find prefreezing and they present evidence for freezing of a surface layer occurring via the KTHNY mechanism. Also, see Ref. [22] for more recent work on walls with much weaker attractions for the particles.

The studies of Gubbins and co-workers used the Steele potential [23] for methane molecules interacting with graphite. At methane's triple point this potential has the large well depth of $-15 k T$. Our surface potential is similar to the Steele potential and has this well depth at $\epsilon_{W S} / \epsilon=26$. This is off the right-hand side of Fig. 1, and so corresponds to a surface at which prefreezing occurs well above the triple point and so the bulk crystal forms immediately the triple-point temperature is crossed. Thus, we predict that liquid methane cannot be supercooled in contact with a graphite surface.

The crystallization of bulk methane at the surface of graphite has not been studied, as far as we are aware. However Castro et al. [24] have studied a methane layer approximately 5.5 molecules thick on the surface of graphite. On cooling the methane layer they found evidence of prefreezing. This is consistent with the results of both Gubbins and co-workers, and ourselves. Castro et al.'s sample is not completely crystalline at the triple point. Again this is in agreement with our simulations. We studied a layer that is 10 molecules thick when completely liquid, against a wall with $\epsilon_{W S} / \epsilon=26$. At the triple point we found a crystal layer 7 molecules thick with a liquid layer approximately 2 molecules thick on top, between the crystal and vapor phases. A snapshot of such a wetting layer is shown in Fig. 3(a).

\section{CRYSTALLIZATION OF THE BULK PHASE}

Having located the surface phase transition and examined its kinetics we will turn to consider the nucleation of the bulk crystal phase. The first point is that once crystal layers have formed at the surface the number of layers can grow as the temperature decreases without crossing a barrier-changing the number of layers is not an activated process. From Fig. 1, we see that at the triple point crystallization at the surface is spontaneous at $\epsilon_{W S} / \epsilon=11$. Thus, for wall attractions stronger than this, even on rapid cooling crystallization occurs at the surface above the triple point and as the triple point temperature is crossed the crystalline layer simply grows into the bulk. Thus, here the nucleation barrier that is crossed on cooling is to prefreezing not freezing.

At the triple point a nucleation barrier is present for all $\epsilon_{W S} / \epsilon<11$; it is still substantial at $\epsilon_{W S} / \epsilon=7$, which is where the wetting transition occurs. This is consistent with studies of hard spheres at a hard wall [3-5].

Auer and Frenkel [5] studied hard spheres at a hard wall at a chemical potential difference between the crystal and fluid phases of $\Delta \mu=-0.05 k T$. Now, at the approximately constant (low) pressure of our system we can obtain an estimate of the chemical potential difference as a function of temperature from $\Delta \mu / k T=\lambda\left(T^{*}-T_{T P}^{*}\right) / T_{T P}^{*}$ [25]. Here $\lambda$ $=1.74[25]$ is the ratio between the enthalpy of fusion and $k T$ at the triple point. Thus in our case $\Delta \mu / k T=-0.05$ at $T^{*}$ $=0.63$. Auer and Frenkel find a nucleation barrier of $17 k T$ 
and we estimate that our barrier is approximately this large at $T^{*}=0.63$ for a wall attraction of $\epsilon_{W S} / \epsilon \approx 10$. Now, Dijkstra [3] finds hysteresis in the hard-sphere system up to a $\Delta \mu / k T \approx 0.1$. This chemical potential difference occurs at $T^{*}=0.69$ in our system. From Fig. 1, we see that for $\epsilon_{W S} / \epsilon$ $\$ 10$ the top of the hysteresis loop is quite close to $T^{*}$ $=0.69$. Thus, we conclude that hard spheres at a hard wall show similar behavior to our system with $\epsilon_{W S} / \epsilon \approx 10$.

To see when nucleation of the bulk crystal becomes spontaneous, at fixed temperature we varied $\epsilon_{W S} / \epsilon$ until nucleation became rapid. These direct simulations were performed in an $L=15 \sigma$ simulation box and the results are plotted in Fig. 1. We see, for example, that at a supercooling of $T^{*}$ $-T_{T P}^{*}=-0.1$ nucleation becomes rapid at $\epsilon_{W S} / \epsilon=6$. We have also used the forward flux sampling (FFS) algorithm of Allen and co-workers $[26,27]$ to calculate nucleation rates at the surface, as we did for homogeneous nucleation in earlier work [11].

In this earlier work, we found that the rate of homogeneous nucleation (away from a surface) was $10^{-13 \pm 1} \sigma^{-3}$ cycle $^{-1}$ at a temperature $T^{*}=0.45$. As nucleation becomes spontaneous at temperatures $T^{*} \leq 0.5$ for all wall attractions $\epsilon_{W S} / \epsilon \geq 4$ we see that for all but very weakly attractive walls heterogeneous nucleation at a wall dominates homogeneous nucleation.

From the FFS simulations we were able to obtain both nucleation rates and configurations of nuclei near the top of the barrier. The FFS simulations were performed in a simulation box with $L=25 \sigma$. At $T^{*}=0.55$ and $\epsilon_{W S} / \epsilon=5$ we find a nucleation rate of $10^{-14 \pm 1} \sigma^{-2}$ cycle $^{-1}$ at the surface. For the higher temperature of $T^{*}=0.6$ and wall attraction of $\epsilon_{W S} / \epsilon$ $=7$ the rate is $10^{-16 \pm 2} \sigma^{-2}$ cycle ${ }^{-1}$. For both sets of conditions, nuclei near the top of the barrier are shown in Fig. 4, and in the supplementary information [28], we include movies showing nucleation and growth. We see that for wall attractions below wetting $\left(\epsilon_{W S} / \epsilon=5\right)$ the nucleus is consistently thicker than for a wall attraction approximately at wetting $\left(\epsilon_{W S} / \epsilon=7\right)$. Figure 4 shows this near the top of the barrier while in the movies we see that at for the more strongly attractive wall the nucleus is flatter both before and after the barrier has been crossed. At the more attractive surface the nucleus has a larger area in contact with the surface. As $\epsilon_{W S}$ is further increased, then nucleation occurs with flatter and flatter nuclei. Also, nucleation occurs at higher and higher temperatures until it occurs above $T_{T P}^{*}$. It is then a surface phase that is nucleating. However, as in earlier work [17], we find no evidence for a sudden change in the nucleus or nucleation rate as the bulk transition is crossed. Eventually however, the crystalline fluctuations become essentially twodimensional, as seen in Fig. 4(a).

\section{CONCLUSION}

To conclude, here we have studied heterogeneous nucleation of a crystal at a flat surface. Crystallization is a common and important phenomenon and generically crystals are expected to nucleate at surfaces that attract the molecules, because there the nucleation barrier is lowest. For relatively weak attractions we find behavior that is qualitatively in agreement with classical nucleation theory $[1,2]$. As the attraction strength grows we reach the point where the crystal wets the liquid-wall interface. At wetting classical nucleation theory predicts the nucleation barrier becomes zero $[1,2]$. This is not the case, instead we show that there are still substantial barriers at $10 \%$ under cooling for a wall interacting with the wetting wall strength. The nucleus however is starting to take on a more two-dimensional appearance. As the wall strength increases the barrier height drops continuously and the nucleus continuously becomes more two dimensional. Then nucleation is occurring above the triple point and is of a new surface not a bulk phase. Classical nucleation theory completely misses the surface phase transition, causing this theory to fail. Once the surface crystal phase has nucleated then there is no barrier to bulk crystallization.

Our observations that surface behavior can control the kinetics of bulk crystallization should be generic. In this paper we have considered two reference systems: hard spheres at a hard wall and methane at a graphite wall. How crystallization occurs in these cases is very different even though in both systems the crystal phase wets the wall-liquid interface. So, to understand bulk crystallization in these systems and indeed in any system in which heterogeneous crystallization occurs, we must consider the surface behavior.

\section{ACKNOWLEDGMENTS}

It is a pleasure to thank S. M. Clarke, D. Frenkel, and J. A. van Meel for discussions. The authors would also like to thank EPSRC and the HPC-Europa program for support of AJP.
[1] R. P. Sear, J. Phys.: Condens. Matter 19, 033101 (2007).

[2] P. G. Debenedetti, Metastable Liquids (Princeton University Press, Princeton, 1996).

[3] M. Dijkstra, Phys. Rev. Lett. 93, 108303 (2004).

[4] B. B. Laird and R. L. Davidchack, J. Phys. Chem. C 111, 15952 (2007).

[5] S. Auer and D. Frenkel, Phys. Rev. Lett. 91, 015703 (2003).

[6] M. Miyahara and K. E. Gubbins, J. Chem. Phys. 106, 2865a (1997).
[7] R. Radhakrishnan and K. E. Gubbins, Mol. Phys. 96, 8 (1999).

[8] R. Radhakrishnan, K. E. Gubbins, and M. SliwinskaBartkowiak, Phys. Rev. Lett. 89, 076101 (2002).

[9] R. Radhakrishnan, K. E. Gubbins, and M. SliwinskaBartkowiak, J. Chem. Phys. 116, 1147 (2002).

[10] D. Frenkel and B. Smit, Understanding Molecular Simulation (Academic, New York, 2002).

[11] J. A. van Meel, A. J. Page, R. P. Sear, and D. Frenkel, J. Chem. Phys. 129, 204505 (2008). 
[12] P. J. Steinhardt, D. R. Nelson, and M. Ronchetti, Phys. Rev. B 28, 784 (1983).

[13] S. Auer and D. Frenkel, J. Chem. Phys. 120, 3015 (2004).

[14] E. Mendez-Villuendas and R. K. Bowles, Phys. Rev. Lett. 98, 185503 (2007); 99, 159901(E) (2007).

[15] The cutoff for a pair of molecules to be considered nearest neighbors is $1.5 \sigma$, and a crystal "link" is taken to exist between neighbors $i$ and $j$ if the product $\mathbf{q}_{6}(i) . \mathbf{q}_{6}(j) \geq 0.65$. See Refs. [11-13] for further details.

[16] R. Ohnesorge, H. Löwen, and H. Wagner, Phys. Rev. E 50, 4801 (1994).

[17] R. P. Sear, J. Chem. Phys. 129, 164510 (2008).

[18] D. Bonn, H. Kelley, and G. H. Wegdam, Phys. Rev. Lett. 69, 1975 (1992).

[19] D. Bonn and D. Ross, Rep. Prog. Phys. 64, 1085 (2001).

[20] T. H. Zhang and X. Y. Lui, J. Am. Chem. Soc. 129, 13520 (2007).

[21] K. J. Strandburg, Rev. Mod. Phys. 60, 161 (1988).

[22] N. Gribova, H. Maleki, A. Arnold, C. Holm, and T. Schilling, e-print arXiv:0902.3344.
[23] W. A. Steele, The Interaction of Gases with Solid Surfaces (Pergamon, Oxford, 1974).

[24] M. A. Castro, S. M. Clarke, A. Inaba, and R. K. Thomas, J. Phys. Chem. B 101, 8878 (1997).

[25] H. Reiss, P. Mirabell, and R. L. Whetten, J. Phys. Chem. 92, 7241 (1988).

[26] R. J. Allen, P. B. Warren, and P. R. ten Wolde, Phys. Rev. Lett. 94, 018104 (2005).

[27] R. J. Allen, D. Frenkel, and P. R. ten Wolde, J. Chem. Phys. 124, 024102 (2006).

[28] See EPAPS Document No. E-PLEEE8-80-070909 for movies created with snapshots from successive interfaces taken from the FFS simulation runs. The FFS runs are performed at $T^{*}$ $=0.6$ at surfaces interacting with $\epsilon_{W S} / \epsilon=5$ and $\epsilon_{W S} / \epsilon=7$. Only the largest cluster of crystalline particles is shown. The surface directly to the left of the cluster is not shown. For more information on EPAPS, see http://www.aip.org/pubservs/ epaps.html. 